\title{
Inverse design of dispersion compensating optical fiber using topology optimization
}

\author{
Riishede, Jesper; Sigmund, Ole
}

Published in:

Journal of the Optical Society of America - B - Optical Physics

Link to article, DOI:

10.1364/JOSAB.25.000088

Publication date:

2008

Link back to DTU Orbit

Citation (APA):

Riishede, J., \& Sigmund, O. (2008). Inverse design of dispersion compensating optical fiber using topology optimization. Journal of the Optical Society of America - B - Optical Physics, 25(1), 88-97.

https://doi.org/10.1364/JOSAB.25.000088

\section{General rights}

Copyright and moral rights for the publications made accessible in the public portal are retained by the authors and/or other copyright owners and it is a condition of accessing publications that users recognise and abide by the legal requirements associated with these rights.

- Users may download and print one copy of any publication from the public portal for the purpose of private study or research.

- You may not further distribute the material or use it for any profit-making activity or commercial gain

- You may freely distribute the URL identifying the publication in the public portal

If you believe that this document breaches copyright please contact us providing details, and we will remove access to the work immediately and investigate your claim. 


\title{
Inverse Design of Dispersion Compensating Optical Fibres Using Topology Optimization
}

\author{
Jesper Riishede and Ole Sigmund* \\ Department of Mechanical Engineering, Technical University of Denmark, Niels Koppel's \\ Allé, Building 404, DK-2800 Kgs. Lyngby, Denmark \\ *Corresponding author: sigmund@mek.dtu.dk
}

In this paper, we present a new numerical method for designing dispersion compensating optical fibres. The method is based on solving the Helmholtz wave equation with a finite-difference modesolver, and uses topology optimization combined with a regularization filter for the design of the refractive index profile. We illustrate the applicability of the proposed method through numerical examples, and furthermore address the problem of keeping the optimized design single-moded by including a single-mode constraint in the optimization problem. (c) 2007 Optical Society of America

OCIS codes: 000.3860, 000.4430, 060.2280, 060.2340, 060.2430

\section{Introduction}

In modern optical communication systems operating at $10 \mathrm{Gbit} / \mathrm{s}$ and above, the use of dispersion compensating fibres (DCFs) is a fundamental requirement for obtaining long transmission lengths without using periodic regeneration of the signal [1]. This requirement stems from the fact that optical pulses broaden as they propagate along an optical fibre, which distorts the bit-information of the signal and thereby makes it difficult to detect the correct signal at the receiver end of the communication system.

From a theoretical point of view, dispersion in optical fibres occurs because the group velocity of the propagating pulses varies as a function of the wavelength. Therefore, the fast spectral components of the pulse will outrun the slow spectral components, and thus give rise to broadening of the pulses. Dispersion is generally divided into two individual contributions, known as material dispersion and waveguide dispersion [2]. Material dispersion is related to the wavelength dependency of the refractive index of silica $\left(\mathrm{SiO}_{2}\right)$ glass, which is the host material of optical fibres used for transmission purposes. Waveguide dispersion, on the other 
hand, is related to the fact that the field distribution of an optical mode changes with the wavelength. This corresponds to a change in the way the electro-magnetic field at a specific wavelength propagates along the fibre, and it therefore modifies the group velocity and gives rise to dispersion.

A possible way to circumvent the problem of dispersion induced pulse broadening is to use DCFs. The basic idea behind a DCF is to modify the fibre design to obtain negative waveguide dispersion that may be used to compensate for the positive material dispersion of $\mathrm{SiO}_{2}$-glass. Typically, the DCFs are designed to have a large negative dispersion coefficient such that one meter of DCF can compensate for several meters of transmission fibre. In the early DCF designs, the large negative dispersion was obtained by using a core region with a small diameter and a large refractive index [3]. However, this simple design does not offer the possibility of controlling the dispersion slope, which is important in modern DWDM-systems where dispersion compensation is required at several wavelengths simultaneously. Therefore, today's DCFs are based on a tri-clad fibre design consisting of a raised index core surrounded by a deeply depressed cladding region which is again surrounded by a lightly raised index ring [1]. As desired, this tri-clad design offers a large negative dispersion, while providing large design freedom for the dispersion slope as well. Tri-clad fibre designs are usually designed to be single-moded, however, in recent works it has been suggested that extremely high negative dispersion can be obtained by special index profiles that support two supermodes $[4,5,6]$. Strong spatial interaction of the supermodes with varying wavelength results in the extremal behavior. Due to the intricate coupling between the supermodes, however, the fibres must be absolutely homogeneous on length-scales exceeding the beating length (order of kilometers), hence manufacturing issues may limit their practical application.

Clearly, the task of finding a proper refractive index profile is an issue of central importance in the design of dispersion compensating fibres. In this paper, we investigate the possibility of using topology optimization for the design of the index-profile. Topology optimization is a numerical method that has been developed within the area of solid mechanics, and has primarily been used for problems of finding the stiffest design using a limited amount of material $[7,8]$. Over the last couple of years the method has spread to other branches of engineering and it is today finding use in such diverse areas as MEMS [9], fluid mechanics [10] and optics. In particular, the recent work by Jensen et. al. on the design of low loss bends in photonic crystal waveguides has created some attention within the optical community $[11,12,13]$.

The idea of using topology optimization for inverse design of DCFs has, to the best of our knowledge, not been reported previously in the literature. In fact, there does not appear to be a tradition of using advanced optimization methods for designing traditional, step-index fibre based DCFs. This is typically because, the number of design variables, i.e. core radius 
and refractive index, is so small that the parameter space can be mapped out entirely [3]. Recently, however, there has been an increasing interest in investigating the potential of using microstructured optical fibres for dispersion compensation. These fibres typically consists of a pure silica core region surrounded by an array of air holes arranged in a hexagonal pattern [14]. In this case, the hole spacing or the diameter of individual air holes may be modified in order to tailor the dispersion properties, and there exists a couple of examples where genetic optimization algorithms have been used for this purpose $[15,16]$.

One of the main advantages of using topology optimization is its ability to find optimized geometries which would not appear from a design approach based on human intuition. This property is caused by the fact that the design is not restricted to any particular shape or overall geometry. Similarly, the aim of the design approach presented here has been to obtain a method for finding waveguide structures for dispersion compensation, where the refractive index distribution is allowed to vary freely over a given design domain. Hence, the optimized design could, for instance, be a microstructured fibre where the air holes or doped index regions are not necessarily circular or not placed in a strict hexagonal pattern.

In the practical implementation, however, it has so far proven to be necessary to apply a few restrictions to the design process, for instance to ensure sufficient confinement of the optical field in the center of the design domain, which is found to give rise to waveguides structures with a complete or nearly cylindrical symmetry. The work presented here, should therefore be seen as a first step of deriving and employing the fundamental theory of a more advanced optimization algorithm for the purpose of designing dispersion properties of optical waveguides. We would also like to emphasize that the aim of this paper is to suggest a method for systematic design of DCFs. At present we do not intend to design a fibre with extremal and record breaking properties but rather we intend to demonstrate that the proposed synthesis method works on a standard single-moded DCF problem. Finally, we note that even though the optimized designs presented in this paper end up having circular symmetry, we define the design domain to be a square domain. In this way, we do not restrict the optimal design to have certain symmetries and we keep the option open for extending our software to high contrast cases that allow for the formation of holey crystal fibers that do not necessarily posses circular symmetry.

The remainder of the paper is organized as follows. In section 2 an introduction to the basic concepts of topology optimization and dispersion in optical fibres is given. Section 3 holds a description of some of the numerical considerations of the implementation of the design algorithms, while section 4 is devoted to a presentation of the numerical results obtained for a DCF that is optimized for three closely spaced wavelengths. In section 5 , the idea of introducing a single-mode constraint to the optimization process is addressed, and finally section 6 holds a conclusion about the presented work. 


\section{Theory of Topology Optimization and Dispersion}

\section{A. The Topology Optimization Method}

Topology optimization is a numerical method for finding improved structures and geometries with respect to a chosen design objective. The method is based on discretizing a physical problem, typically described by a PDE, and solving it iteratively, while performing consecutive small modifications to the material distribution and thereby indirectly the geometry. From a general point of view, topology optimization consists of many different computational steps which are listed schematically in figure 1 . The different steps will be outlined in detail in the following:

First, an initial design guess is made by assigning a material value to those grid points that make up the design domain of the considered optimization problem. Then, the PDE describing the considered physical problem is solved, and the initial value of the objective function is calculated. The objective function is a scalar quantity that expresses the state of the optimization process, and which needs to be minimized by modifying the design variables in order to solve the optimization problem.

A requirement for performing efficient updates of the design variables is to have some knowledge of how the objective function varies with the individual design variables. The gradient of the objective function, commonly referred to as the sensitivity, is calculated in the sensitivity-analysis step. Since the objective function is a global quantity, i.e. defined over the entire design domain, the sensitivity analysis can be calculated very efficiently using an analytical approach called the adjoint method.

Once the sensitivities have been calculated, it is a common approach in topology optimization to do a regularization of the design problem for example by filtering the sensitivities [17]. This is done in order to reduce the mesh dependency of the optimization process, which means that the discretization of the PDE can be refined without affecting the optimized design significantly. Using the filtered sensitivities, the optimization of the objective function is performed, and the design variables are updated. Typically, the optimization step is carried out using mathematical programming tools, where it is possible to include one or moredesign constraints in the optimization process.

After the optimization step, the entire process is repeated until a desired convergence criterion is met. As indicated in figure 1 the convergence check, and a possible exit of the optimization loop, is made after the calculation of the objective function, where it is possible to compare the objective function of the newly optimized design with previous results.

\section{B. Modelling of Dispersion Properties}

In the present optimization problem, the aim is to modify the refractive index distribution of an optical fibre in order to optimize the dispersion properties. The dispersion parameter, 
$D$, is defined as:

$$
D=-\frac{\lambda}{c} \frac{d^{2} n_{\mathrm{eff}}}{d \lambda^{2}}
$$

Here, $\lambda$ is the free space wavelength, $\mathrm{c}$ is the speed of light and $n_{\text {eff }}$ is the so called effective index of the guided mode. The effective index is related to the propagation constant, $\beta$, and the wavenumber, $k=2 \pi / \lambda$ as:

$$
n_{\mathrm{eff}}=\frac{\beta}{k}
$$

and may be interpreted as the average refractive index seen by the optical field.

The preferred way of modelling dispersion properties in optical fibres is to use modal analysis to find the propagation constant, $\beta$. Here, we choose a mathematical formulation based on solving the scalar Helmholtz wave equation for a given index distribution $n(x, y)$ :

$$
\nabla_{t}^{2} \psi(x, y)+n^{2}(x, y) k_{0}^{2} \psi(x, y)=\beta^{2} \psi(x, y)
$$

As seen, Eq. (3) is an eigenvalue problem, where $\beta$ is the square-root of the eigenvalue and the field distribution of the optical mode, $\psi(x, y)$, is obtained from the corresponding eigenvector. Strictly speaking, the propagation of light in optical fibres is governed by Maxwell's equations, which generally need to be solved using full-vectorial methods. However, the scalar Helmholtz equation is a commonly used approximation to Maxwell's equations which is known to be valid in situations where the index-contrast of the considered waveguide geometry is small. In the current situation where we are considering optical fibres made from a combination of pure and $\mathrm{Ge}$-doped $\mathrm{SiO}_{2}$-glass, the obtainable index contrasts are typically in the order of a few percent, thus making the use of the scalar approximation feasible.

In this paper, the modelling of the scalar Helmholtz equation is carried out using a simple finite-difference modesolver [18]. The basic idea of the method consist in discretizing the optical field, $\psi(x, y)$, and the refractive index distribution, $n(x, y)$, and approximating the partial derivatives by finite-differences. For the scalar Helmholtz equation in Eq. (3), we choose to approximate the partial second order derivative by a three-point finite-difference scheme of the form:

$$
\begin{array}{r}
\nabla^{2} \psi(x, y) \approx \frac{\psi(x-\Delta x, y)-2 \psi(x, y)+\psi(x+\Delta x, y)}{(\Delta x)^{2}}+ \\
\frac{\psi(x, y-\Delta y)-2 \psi(x, y)+\psi(x, y+\Delta y)}{(\Delta y)^{2}}
\end{array}
$$

This approach gives rise to one linear equation for each grid point and by combining all these equations the Helmholtz equation is transformed into an eigenvalue problem of the form:

$$
\Phi \psi=\beta^{2} \psi
$$

where the matrix, $\Phi$, is here referred to as the discretization matrix of the finite-difference problem. In the following consider a calculation domain discretized in $m$ by $n$ grid points 
in the $\mathrm{x}$ - and $\mathrm{y}$-direction, respectively, where the grid points are numbered in consecutive order from 1 to $m \times n$. Furthermore, assume that the grid points are equidistantly spaced and have the coordinates $x_{i}$ and $y_{i}$, with $i$ being the number of the grid point. In this case, $\Phi$, becomes a square matrix where the elements are given as:

$$
\Phi(i, j)= \begin{cases}\frac{-4}{d^{2}}+n\left(x_{i}, y_{i}\right)^{2} k_{0}^{2} & ; \text { for } i=j \\ \frac{1}{d^{2}} & ; \text { for } i, j \text { nearest neighbours } \\ 0 & ; \text { otherwise }\end{cases}
$$

Here, $d$ is the grid spacing, $n\left(x_{i}, y_{i}\right)$ is the discretized representation of the refractive index distribution, and $\mathrm{i}, \mathrm{j}$ are integers running from 1 to $m \times n$. Furthermore, the term "nearest neighbors" refers to neighboring grid points in the discretized calculation domain. As seen from the definition in Eq. (6), the $\Phi$-matrix is symmetric and highly sparse, which may be utilized to obtain a cheap storage and efficient solution of the problem.

\section{C. Objective Function and Sensitivity Analysis}

The aim of the optimization problem is to modify the refractive index distribution of the optical fibre such that the dispersion matches a predefined value at one or several wavelengths simultaneously. For this purpose, we formulate the design problem as a task of minimizing an objective function of the form:

$$
f=\sum_{i=1}^{N} \frac{\left(D_{i}^{*}-D_{i}\right)^{2}}{\left(D_{i}^{*}\right)^{2}}
$$

Here, $N$ is the number of wavelengths at which the index distribution is optimized, and $D_{i}^{*}$ and $D_{i}$ are the target value and the calculated value of the dispersion coefficient at the $i$ 'th wavelength, respectively.

From Eq. (1) it is known that the dispersion coefficient is proportional to the second derivative of the effective index, $n_{\text {eff }}$, which is again related to the eigenvalue, $\beta^{2}$, of the Helmholtz wave equation. Thus, in a straight forward approach, the calculation of the sensitivity of $f$ with respect to the design variable will give rise to a problem involving a third order derivative of an eigenvalue. In topology optimization problems, the analytical sensitivity analysis is typically found using the adjoint method [8], which ensures that the sensitivity analysis can be carried out using a very small numerical effort. Unfortunately, calculating the third-order derivative of an eigenvalue using this approach is not a trivial task. Thus, to work around this problem we instead choose a simplified approach, where the dispersion coefficient is expressed by a three point finite-difference scheme:

$$
D \approx-\frac{\lambda}{c} \frac{n_{\mathrm{eff}, 1}-2 n_{\mathrm{eff}, 2}+n_{\mathrm{eff}, 3}}{(\Delta \lambda)^{2}}
$$


Here, we find the second derivative at the wavelength $\lambda$ by calculating the effective index, $n_{\mathrm{eff}}$, at three different wavelengths separated by $\Delta \lambda$. By using the finite-difference approximation to the dispersion, the sensitivity analysis is instead reduced to a problem of finding a first order derivative of an eigenvalue, which is known to be a simple problem. However, this advantage comes at the cost of having to solve three eigenvalue problems for each dispersion calculation so computation time increases accordingly.

In order to represent the topology of the fibre structure, we introduce a design variable, $\gamma_{i}$, having a value between 0 and 1 in each grid point of the design domain. The relation between the design variable and the refractive index is given by the expression:

$$
n_{i}=n_{\min }+\gamma_{i}\left(n_{\max }-n_{\min }\right)
$$

where $n_{\max }$ and $n_{\min }$ represent the maximum and minimum value of the refractive index, respectively. The sensitivity of the objective function with respect to the i'th design variable, $\gamma_{i}$, may therefore be written as:

$$
\frac{\partial f}{\partial \gamma_{i}}=2\left(n_{\max }-n_{\min }\right) \sum_{j=1}^{N} \frac{\left(D_{j}^{*}-D_{j}\right)}{\left(D_{j}^{*}\right)^{2}} \frac{\partial D_{j}}{\partial n_{i}}
$$

The difficult part of evaluating the expression in Eq. (10) consists in determining the derivative of the dispersion coefficient with respect to the refractive index in the $i$ 'th grid point $\partial D_{j} / \partial n_{i}$. By differentiating the finite-difference representation of the dispersion coefficient in Eq. (8) we are faced with the task of finding the first derivative of the effective index with respect to $n_{i}$. From the definition of $n_{\text {eff }}$ in Eq. (2), the first order derivative of the effective index may be written as:

$$
\frac{\partial n_{\mathrm{eff}}}{\partial n_{i}}=\frac{\partial\left(\sqrt{\beta^{2}} / k_{0}\right)}{\partial n_{i}}=\frac{1}{k_{0}^{2}} \frac{1}{2 n_{\mathrm{eff}}} \frac{\partial\left(\beta^{2}\right)}{\partial n_{i}}
$$

where the last term corresponds to the derivative of the eigenvalue, $\beta^{2}$, of the Helmholtz wave equation.

The derivative of an eigenvalue with respect to a design variable can be obtained almost for free, once the eigenvalue and the eigenvector have been calculated. By multiplying Eq. (3) with $\psi^{T}$ and taking the derivative, the following expression is obtained:

$$
\frac{\partial \psi^{T}}{\partial n_{i}} \Phi \psi+\psi^{T} \frac{\partial \Phi}{\partial n_{i}} \psi+\psi^{T} \Phi \frac{\partial \psi}{\partial n_{i}}=\frac{\partial\left(\beta^{2}\right)}{\partial n_{i}} \psi^{T} \psi+2 \beta^{2} \psi^{T} \frac{\partial \psi}{\partial n_{i}}
$$

Now, by normalizing the eigenvectors $\left(\psi^{T} \psi=1\right.$ ), use of Eq. (5) and utilizing the that $\Phi$ is symmetric, Eq. (12) may be reduced to:

$$
\frac{\partial\left(\beta^{2}\right)}{\partial n_{i}}=\psi^{T} \frac{\partial \Phi}{\partial n_{i}} \psi
$$


From the definition of the $\Phi$-matrix in Eq. (3) it is seen the that the refractive index of the i'th grid point, $n_{i}$, only appears in the diagonal element of the i'th row. Thus, the derivative of the eigenvalue with respect to $n_{i}$ is simply given as:

$$
\frac{\partial\left(\beta^{2}\right)}{\partial n_{i}}=\psi^{T} \frac{\partial \Phi}{\partial n_{i}} \psi=2 n_{i} k_{0}^{2} \psi_{i}^{2}
$$

where $\psi_{i}$ is the value of the field distribution in the i'th grid point. If this result is combined with the three point formula in Eq. (8), the following expression for the derivative of the dispersion is obtained:

$$
\frac{\partial D}{\partial n_{i}}=-\frac{\lambda}{c} \frac{n_{i}}{(\Delta \lambda)^{2}}\left(\frac{\psi_{1, i}^{2}}{n_{\mathrm{eff}, 1}}-2 \frac{\psi_{2, i}^{2}}{n_{\mathrm{eff}, 2}}+\frac{\psi_{3, i}^{2}}{n_{\mathrm{eff}, 3}}\right)
$$

Here, it is emphasized that the effective indices and eigenvectors are calculated at three different wavelengths, where $\lambda$ refers to the center wavelength. Inserting the expression in 15 into Eq. (10) yields the final expression for the sensitivity of the dispersion with respect to the design variable, $\gamma_{i}$. In conclusion, the sensitivities can be obtained by virtually no added cost when the original Helmholtz problem in Eq. (3) already has been solved.

\section{D. Density Filter}

To reduce the mesh-dependency of the optimized solution, a filtering method is included in the optimization process. There exists several filtering approaches, see [17] and references therein, but here we choose to use a density filtering technique, as suggested in refs. [19, 20]. The advantage of using density filtering is that it introduces a limitation on the local gradient of the material properties, and thus acts to smooth out the topology ensuring meshindependent and manufacturable optimized designs.

The basic idea of the density filtering technique consists in introducing a new design parameter, $\tilde{\gamma}_{j}$, that represents a weighted average of the design variables, $\gamma_{i}$, within a certain neighborhood. The averaged design parameter is defined as:

$$
\tilde{\gamma}_{j}=\frac{\sum_{i=1}^{N} \gamma_{i} s_{i}(j)}{\sum_{i=1}^{N} s_{i}(j)}
$$

where $s(j)$ is a filter function (window) centered in the $j$ 'th grid point, that selects the design variables in the grid points that fall within the area of the filter. Figure 2 shows three examples of the shape of the filter window for increasing values of the filter width, $w$. As seen in the figure, the design variables are sampled with a weight of 1 inside the filter and zero outside the filter. We only use filters with an uneven value of the filter width in order to make the filtering symmetric with respect to the central grid point. 
The averaged design variable, $\tilde{\gamma}_{j}$, is used to represent the physical topology of the considered problem, which in our case means that the refractive index of the waveguide geometry is now related to $\tilde{\gamma}_{j}$ as:

$$
n_{j}=n_{\min }+\tilde{\gamma}_{j}\left(n_{\max }-n_{\min }\right)
$$

The optimization, however, is still carried out with respect to the original design variables. Therefore, the expression for the sensitivity with respect to $\gamma_{i}$ has to be reformulated. To derive the new expression for the sensitivity, it has to be emphasized that the sensitivity given in Eq. (10) was derived for a design variable being proportional to the refractive index distribution, and therefore corresponds to the sensitivity of the averaged design variable, $\tilde{\gamma}_{j}$. Finally, the new sensitivities can be obtained by the chain rule as:

$$
\frac{\partial f}{\partial \gamma_{i}}=\sum_{j=1}^{N} \frac{\partial f}{\partial \tilde{\gamma}_{j}} \frac{\partial \tilde{\gamma}_{j}}{\partial \gamma_{i}} s_{j}(i)=\sum_{j=1}^{N} \frac{\partial f}{\partial \tilde{\gamma}_{j}} \frac{s_{j}(i)}{M(i)}
$$

Here, $M(i)$ is the number of non-zero grid points in the filter and $\frac{\partial f}{\partial \tilde{\gamma}_{i}}$ is the sensitivity calculated from Eqs. (10) and (15).

\section{Numerical Considerations}

The actual numerical implementation of the optimization algorithm, i.e. the modesolver, the sensitivity analysis and the mathematical programming steps, have been carried out entirely in Matlab. For the finite-difference modesolver, we have chosen to enforce mirror symmetry of the waveguide geometry in both the $\mathrm{x}$ - and y-direction, in order to reduce the computational requirements of solving the Helmholtz wave equation. The computation is therefore only performed using the upper left quadrant of the fibre geometry. On the lower and right boundaries of the reduced calculation domain we impose two different types of boundary conditions; one which assumes that that field, $\psi(x, y)$, is unchanged on the other side of the boundary (i.e. a Neuman boundary condition) and a second which assumes a change of sign in the field amplitude (i.e. a Dirichlet boundary condition). Thereby it is possible to control the basic symmetry of the obtained field solution, i.e. if we are modelling an even LP01-mode or an uneven LP11-mode. On the right and upper edge of the calculation domain, a Dirichlet boundary condition that sets the field to zero is applied. The Dirichlet boundary condition is a good representation of the actual behavior of the optical field, as long as the guided mode is well confined to the core region of the waveguide.

In the case of the optimization step, we have chosen to use the Method of Moving Asymptotes (MMA), which is one of the most well established mathematical programming methods within the field of topology optimization [21]. The method allows the optimization to be subjected to one or several constraints. 


\section{Dispersion Optimization at Three Wavelengths}

Using the theory derived in the previous sections we now turn to the problem optimizing the refractive index profile of a DCF. The aim of the optimization is to obtain a DCF that has a compensation ratio of 5 compared to a standard non-dispersion shifted transmission fibre operating at a wavelength around $1.55 \mu \mathrm{m}$.

First, the dispersion properties of the transmission fibre are found. The fibre parameters are chosen to resemble that of an SMF-28 single-mode fibre [22]. Hence, the fibre has a simple step-index profile with a core diameter of $d=8.2 \mu \mathrm{m}$ and an index step, $\Delta n=0.36 \%$ compared to the cladding which is assumed to consist of pure silica. In order to include the effect of the material dispersion, the refractive index of silica is determined from the Sellmeier polynomial [2]. Using the listed parameters, the step-index fibre is found to have a positive dispersion of $20.5 \mathrm{ps} / \mathrm{km} / \mathrm{nm}$ at $\lambda=1.55 \mu \mathrm{m}$ and a zero dispersion wavelength at approximately $1.27 \mu \mathrm{m}$.

For the optimization of the DCF-design, we choose to discretize the upper left quadrant of the fibre structure in $100 \times 100$ grid points while the width of this domain is set to 50 $\mu m$ in both the $\mathrm{x}$ - and $\mathrm{y}$-direction. A design variable is assigned to each of these grid points in the calculation domain which means that the considered optimization problem consists of 10000 design variables in total. For the optimized design, we wish to obtain both dispersionand dispersion-slope matching around $\lambda=1.55 \mu \mathrm{m}$. Therefore, the dispersion optimization is carried out at three wavelengths, $\lambda_{1}=1.5475 \mu \mathrm{m}, \lambda_{2}=1.55 \mu \mathrm{m}$ and $\lambda_{3}=1.5525 \mu \mathrm{m}$.

To include material dispersion in the optimization of the DCF design, the maximum and minimum value of the refractive index is defined with respect to the refractive index of silica. Here, we set $n_{\max }=n_{\mathrm{SiO}_{2}}+0.015$ and $n_{\min }=n_{\mathrm{SiO}_{2}}-0.005$. For the initial value of the design variables, we choose to set $\gamma_{i}=0.25$, corresponding to the refractive index of $\mathrm{SiO}_{2}$, over the entire calculation domain, apart from a circular area in the center of the domain, where the design variables have a value of 1 . This high-index region is introduced, since the optimized fibre design should have a core region placed in the center of the calculation domain, and we generally also want the field of the DCF to be well confined, in order to avoid influence of the Dirichlet boundary conditions. We have tried to start from a completely flat initial design and use different kinds of constraints, for instance putting a limitation on the effective area of the guided mode, to obtain a design with a core region in the centre. We have concluded, however, that the concept of introducing a high-index area in the initial design is by far the simplest and most effective approach.

By minimizing the objective function of the design problem using a filter width of 5 grid points for the density filter and a diameter of $10 \mu \mathrm{m}$ for the core region of the initial design, we obtain a DCF with the dispersion properties as shown in figure 3. The optimized topology is found after 295 iterations (i.e. FD-calculations). For comparison, figure 3 also 
contains a dispersion curve corresponding to -5 times the dispersion of the reference singlemode transmission fibre. As seen from the figure there is a good agreement between both the dispersion and the dispersion slope at $\lambda=1.55 \mu \mathrm{m}$. This fact is further illustrated by the inset, which shows a close-up of the dispersion curves. Here, it is clearly seen that the optimized DCF design has a complete match of the dispersion at the target wavelength $\lambda_{2}=1.55 \mu \mathrm{m}$, and a small (but negligible) deviation at $\lambda_{1}=1.5475 \mu \mathrm{m}$ and $\lambda_{3}=1.5525 \mu \mathrm{m}$ due to the curvature of the optimized dispersion curve.

Figure 4 shows the spatial distribution of the refractive index of the optimized fibre design. Note that the optimization has only been carried out for the upper left quadrant of the fibre structure, which in this case has been copied to the three other quadrant to show the entire structure. Furthermore, it is emphasized that the refractive index is proportional to the averaged density variable, $\tilde{\gamma}_{j}$, which again is found from the actual design variables, $\gamma_{i}$, by applying the density filter in Eq. (16). As mentioned previously, the fibre structure is seen to have a clear circular symmetry, even though the design variables are allowed to vary freely and create other (square symmetric) shapes and geometries. Even if we start the optimization process with a rectangular core region, the topology converges to a circular design, indicating that the optimal solution indeed is rotational symmetric. To obtain a more detailed view of the topological features of the refractive index distribution, figure 5a shows the index profile of the optimized design through the center axis of the calculation domain. The optimization has been carried out with a density filter width of $\mathrm{w}=5$. To illustrate the effect of using the density filter figure 5b shows the same calculation, where the density filter has been disabled (w ; 1). By comparison it is clearly seen how the density filter acts to smooth out the index profile and removes features on the scale of a single grid point. It should be emphasized that the density filter, and the associated smoothing of the design variables, is not applied as a post processing step but is included directly in the mathematical formulation of the design problem. It is interesting to remark that the objective function of the filtered design (figure 5a) is around 3\% better than for the non-filtered design (figure 5b), eventhough the filtering actually decreases the design space. This indicates that the filtering actually has a smoothening effect on the optimization problem and prevents convergence to local optima.

Considering the shape of the index-profile in figure 5a, it is noted that the optimized design contains a depressed cladding surrounded by a raised index ring, and it therefore has a close resemblance to the traditional tri-clad fibre design used in modern DCFs. This result therefore strongly indicates, the that the traditional tri-clad DCF design appears to be the optimum design approach, since the topology optimization algorithm does not suggest a radically different design.

A more detailed study of the optimized index-profile, reveals that the raised cladding contains two peaks. The outer peak stems from the early stage of the optimization process, 
where the field is not as tightly confined to the core region. As a consequence material is added over an area with a large diameter. From a visual inspection of the optimization process, it is noted that the confinement properties of the fibre are seen to increase rapidly at some point, and as a result material is added close to the core region. This gives rise to the inner peak of the index-profile. Since the field is better confined to the core region in the final design, the outer peak does therefore not have a strong impact on the dispersion properties. The final topology may therefore include features that arises as a consequence of the path of the optimization process. By using a different path for the optimization, e.g. by using a different initial design, a slightly modified topology will be obtained. This example illustrates the important fact, that the inverse problem of designing a index-profile that matches a given dispersion is not unique, i.e. there exists many different topological solutions that solve the optimization problem. The number of possible solution may be reduced by using a density filter, or by introducing a constraint on the optimization as discussed in the following section.

\section{Optimization Including a Single-mode Constraint}

\section{A. Theory}

The results discussed in the previous sections have served to illustrate that the basic idea of using topology optimization for design of dispersion compensating optical fibres is indeed feasible. A general problem with the optimized designs, which have been presented so far, is that they all are severely multi-moded. Considering the optimized fibre geometry in figure $5 \mathrm{a}$ it is seen that modifications to the dispersion properties have mainly been obtained by adding high-index material to the design. This effectively creates an internal cladding region with a diameter of approximately $50 \mu \mathrm{m}$ where higher order modes are allowed to exist. As single mode operation is crucial for a DCF, since it would otherwise add significantly more dispersion to the signal than it is designed to compensate for, it is required to address this issue by introducing some kind of single-mode constraint to obtain truly functional designs. As noted in the introduction, extremely high dispersion can be obtained in multi-moded fibres, however, since our goal is to present a generally applicable design method and not to suggest new and extremal fibre designs, we will in the following stick to the simpler and easier to manufacture single-moded DCFs.

From a theoretical point of view, an optical fibre becomes single-moded when all higher order modes have reached cut-off, i.e. when their respective effective indices fall below the refractive index of the cladding region. This formulation of the single-mode definition is straight forward to adapt for fibre geometries where the cladding region is a well defined area, i.e. a traditional step-index fibre. However, in our present formulation of the optimization problem this is not the case. This stems from the fact that the refractive index is allowed to vary over the entire calculation domain during the optimization which makes it difficult to 
determine an exact value of the refractive index of the cladding region. In order to solve this problem, a modification to the optimization approach is introduced. Instead of performing the optimization over the entire calculation domain, we instead define a circular optimization domain in the centre of the calculation domain. Only grid points that fall within the diameter of the optimization domain, $d_{\text {opt }}$, will be subject to optimization. The refractive index of the remaining part of the calculation domain is assigned a fixed value, $n_{\mathrm{bg}}$, which will serve as the limit determining whether a higher order mode is guided or has reached cut-off.

The optimization problem to be solved is now defined as:

$$
\begin{array}{rrr}
\min _{\gamma_{i}} & f=\sum_{i=1}^{N} \frac{\left(D_{i}^{*}-D_{i}\right)^{2}}{\left(D_{i}^{*}\right)^{2}} \\
\text { s.t. } & n_{\mathrm{eff}_{L P 11}<n_{\mathrm{bg}}} \\
\text { s.t. } & n_{\mathrm{eff}_{L P 01}>n_{\mathrm{bg}}}
\end{array}
$$

The objective function in Eq. (19) is the same as before but as seen, two constraints have been added to the optimization problem. The constraint in Eq. (20) is the single-mode constraint which forces the effective index second-order mode, LP11, below the cut-off value. We have found that the constraint is very efficient at doing this. However it has the side-effect of forcing the effective index of the fundamental mode, LP01, close to the background index as well. This causes the field of the fundamental mode to become strongly delocalized, which affects the accuracy of the solution due to the Dirichlet boundary condition that is applied on the edges of the calculation domain. To counteract this behaviour, it has been necessary to add the constraint in Eq. (21) which forces the effective index of the fundamental mode to stay above the background index.

In order for the MMA optimization algorithm to satisfy the constraints it is necessary to calculate the sensitivity of the constraints with respect to the design variables. For the constraint involving the LP01-mode the sensitivity is given as:

$$
\frac{\partial n_{\mathrm{eff}_{L P 01}}}{\partial n_{i}}=\frac{n_{i}}{n_{\mathrm{eff}}} \psi_{i}^{2}
$$

For simplicity the sensitivity is here shown with respect to the refractive index. In the case of the single-mode constraint, which involves the LP11-mode, the calculation of the sensitivity becomes more complicated. This is due to the fact that the second-order mode in an optical fibre consists of two orthogonal field solutions with the same effective index, i.e. the two LP11-modes represent a degenerate eigensolution. As a result the eigenvectors $\psi_{1}$ and $\psi_{2}$, which represent the field distribution of the modes, may be added to form a new eigenvector:

$$
\psi=c_{1} \psi_{1}+c_{2} \psi_{2}
$$

where $c_{1}$ and $c_{2}$ are arbitrary constants. 
If the new eigenvector is inserted into the expression for the sensitivity in Eq. (22) the following expression is obtained:

$$
\frac{\partial n_{\mathrm{eff}}}{\partial n_{i}}=\left(c_{1}^{2} \psi_{1, i}^{2}+c_{2}^{2} \psi_{2, i}^{2}+2 c_{1} c_{2} \psi_{1, i} \psi_{2, i}\right) \frac{n_{i}}{n_{\mathrm{eff}}}
$$

The result in Eq. (24) illustrates the basic problem of calculating the sensitivity of a degenerate eigenvalue, namely that the value depends on the choice of the constants $c_{1}$ and $c_{2}$. To address this problem, we here follow the approach described in refs. [23, 24]. The basic idea here is to select the extremal values of the span of possible eigenvectors.

First we differentiate the expression in Eq. (24) with respect to $c_{1}$ and $c_{2}$ and set the result equal to zero. This leads to the the following set of linear equations:

$$
\left[\begin{array}{cc}
\frac{n_{i}}{n_{\mathrm{eff}}} \psi_{1, i}^{2} & \frac{n_{i}}{n_{\mathrm{eff}}} \psi_{1, i} \psi_{2, i} \\
\frac{n_{i}}{n_{\mathrm{eff}}} \psi_{1, i} \psi_{2, i} & \frac{n_{i}}{n_{\mathrm{eff}}} \psi_{2, i}^{2}
\end{array}\right]\left\{\begin{array}{c}
c_{1} \\
c_{2}
\end{array}\right\}=\left\{\begin{array}{l}
0 \\
0
\end{array}\right\}
$$

The sensitivities of the two degenerate LP11-modes correspond to the eigenvalues of the matrix in Eq. (25). By solving the eigenvalue problem analytically it is realized that the first of the two eigenvalues is always zero. As a result the sensitivity of the degenerate LP11eigenstate is defined solely be the second eigenvalue of the matrix in Eq. (25) and is given as:

$$
\frac{\partial n_{\mathrm{eff}_{L P 11}}}{\partial n_{i}}=\frac{n_{i}}{n_{\mathrm{eff}}}\left(\psi_{1, i}^{2}+\psi_{2, i}^{2}\right)
$$

By comparison, this expression is seen to have a strong similarity to the expression for the LP01 sensitivity in Eq. (22), only Eq. (26) depends on the field distribution of both polarisations, $\psi_{1}$ and $\psi_{2}$.

\section{B. Optimization}

Having established the methodology for calculating the derivatives of the two constraints in Eqs. (20) and (21), we redo the optimization problem considered in section 3 while applying the two constraints. To recapitulate, the optimization is carried out on a calculation domain (representing the upper left quadrant of the fibre geometry) discretized in $100 \times 100$ grid, uses a width of $w=5$ of the regularization filter and aims at matching the dispersion at three wavelengths, $\lambda_{1}=1.5475 \mu \mathrm{m}, \lambda_{1}=1.5500 \mu \mathrm{m}$ and $\lambda_{3}=1.5525 \mu \mathrm{m}$. In addition, the diameter of the central optimization domain, which is used in the new formulation of the optimization problem, is chosen to $d_{\text {opt }}=20 \mu \mathrm{m}$. This area contains 331 design variables. The fixed refractive index of the background material is set to $n_{b g}=1.4440$, which corresponds to the refractive index of pure silica at $\lambda=1.5475 \mu \mathrm{m}$. By using the refractive index of the lowest optimization wavelength we ensure that the design is single-moded at all considered wavelengths. 
Figure 6 shows a cross-section of the optimized fibre geometry obtained after 306 iterations. Again, the fibre geometry is seen to have the traditional W-profile known from conventional dispersion compensating fibres. Compared to the previous designs shown in figure $5 \mathrm{a}$ a number of differences are observed. First, the part of the new fibre geometry which has been changed during the optimization is seen to be more narrow compared to the previous design. This is a direct consequence of limiting the optimization to only the $20 \mu \mathrm{m}$ wide circular section in the centre of the calculation domain. Secondly, the average magnitude of the refractive index in the raised index ring is found to be small compared to the previous design. As a result only the fundamental mode is found to be guided in the fibre structure which demonstrates that the single-mode constraint is working as expected.

It is generally found that the designs obtained from optimization subject to the singlemode constraint provides an equally good solution, with respect to matching the dispersion at the three target wavelengths, as it is shown in figure 3 for the optimization without constraints. Therefore, adding the two constraints to the optimization process is not seen to affect the quality of the obtained solutions, it only helps the optimization to focus on those parts of the solution space that contain the single-moded solutions.

To provide a better understanding of how the single-mode constraint works, figure 7 shows the development of the effective index against the iteration number during the optimization. The figure contains the effective index for the fundamental mode, LP01, and the secondand third-order mode, LP11 and LP02. All mode indices are found at a wavelength of $\lambda=1.5475 \mu \mathrm{m}$ which corresponds to the lowest wavelength in the optimization. The figure furthermore contains a line representing the refractive index of the background material, $n_{b g}=1.4440$. It is clearly seen how the effective indices of the higher order modes are forced below the refractive index of the background region, thus making the optimized design singlemoded. An additional inset has been included in the figure to illustrate that the effective indices are in fact below the background index also at the end of the optimization.

It should be noted that the optimization only has been performed with a constraint on the LP01- and LP11-mode. The reason for including the LP02-mode in figure 7 as well stems from the fact that the transition from single-mode to multi-mode operation may be governed by the cut-off of the LP02-mode in cases where the fibre has a W-like geometry. By plotting the LP02-mode as well, we are able to monitor that it also satisfies the cut-off condition. Alternatively we could have included a constraint similar to Eq. (26) for the LP02-mode. However, as this has so far not proven to be a requirement for obtaining a single-moded design, and as it would increase the computational demands, we have chosen to omit this constraint.

An important issue to notice about the single-mode constraint is that it requires modelling of the cut-off condition of the higher-order modes. Generally, numerical modelling of the 
cut-off wavelength in a waveguide is difficult. This is because the field becomes strongly delocalized near the cut-off conditions, which means that it requires use of a large calculation domain to avoid that the truncation of the calculation domain influences the solution. In our case we solve the wave equation by using a homogeneous finite-difference discretization, and hence a large expansion of the calculation domain would cause a dramatic increase in the computational demands. However, as the objective of the analysis has not been to present a highly accurate calculation of the cut-off conditions of the waveguide, but rather to provide a proof-of-concept and demonstrate the applicability of the suggested single-mode constraint, we have chosen to accept that the modelling of the cut-off of the higher order modes is (to some extent) affected by the Dirichlet boundary condition.

If the problem of modelling the cut-off conditions accurately is to be addressed, it will require a different modelling strategy than the one applied in this work. One solution could be to use a finite-difference method using a non-homogeneous grid to allow a less detailed discretization of the cladding region. A similar approach could be to use a finite-element method with refined meshing around the core region. Alternatively, one could consider the idea of modelling an actual fibre geometry which is bounded by a finite, circular cladding structure. A detailed study of any of the mentioned approaches is considered beyond the scope of this work, but holds some interesting ideas to be addressed in future work.

\section{Conclusion}

In this paper, we have presented a method for designing dispersion compensating fibres using the topology optimization method. The work is based on solving the scalar Helmholtz wave equation with a finite difference method to determine the fibre properties, while the optimization is performed by applying the Method of Moving Asymptotes. To avoid meshdependent solutions, and to obtain a smoothing of the designs, the method furthermore includes a regularization strategy based on density filtering.

We have shown that the method is capable of designing a fibre geometry that has dispersion properties matching a predefined dispersion curve at a number of wavelengths. The specific aim in this paper has been to design a DCF for compensating the dispersion of a standard SMF28 single-mode fibre at three wavelengths around $1.55 \mu \mathrm{m}$. Such a fibre design was successfully obtained, and its dispersion properties was found to be in excellent agreement with the target dispersion curve both with respect to dispersion magnitude and dispersion slope.

As the optimized designs proved to be multi-moded, which generally is an undesired property for a DCF, a single-mode constraint was included in the optimization problem. The basic purpose of this constraint is to force the effective mode-index of the higher order modes below the cladding index of the structure. This approach has proved to be feasible, thus eliminating 
the problem with multi-moded fibre designs.

It is remarkable that even though the design formulation allows for non-circular designs, we have always ended up with optimized structures with circular symmetry. Hence, for the low index contrast problems which we have been considering in this paper, we could as well have parametrized the design as a radially varying function, however, in future work we are extending the method to high index contrast problems, e.g. photonic crystal fibers, and here we expect optimized structures to be non-circular symmetric including freely varying hole shapes.

Based on the results presented in this paper, we conclude that topology optimization represents an efficient approach for designing dispersion compensating fibers.

\section{Acknowledgements}

The authors are grateful to Professor Niels Asger Mortensen, Technical University of Denmark for fruitful suggestions concerning dispersion compensating fibers. This work received support from the Eurohorcs/ESF European Young Investigator Award (EURYI, www.esf.org/euryi) through the grant "Synthesis and topology optimization of optomechanical systems" and a New Energy and Industrial Technology Development Organization project (NEDO, Japan). 


\section{References}

1. L. Grüner-Nielsen, S. N. Knudsen, B. Edvold, T. Veng, D. Magnussen, C. C. Larsen and H. Damsgaard "Dispersion Compensating Fibres," Optical Fiber Technology 6, 164-180 (2000).

2. G. Agrawal "Fiber-Optic Communication Systems, 2nd ed.," John Wiley \& Sons (1997).

3. A. Bjarklev, T. Rasmussen, O. Lumholt, K. Rottwitt and M. Helmer "Optimal design of single-cladded dispersion-compensating optical fibres," Optics Letters 19, 457-459 (1994).

4. J. L. Auguste, J. M. Blondy, J. Maury, J. Marcou, B. Dussardier, G. Monnom, R. Jindal, K. Thyagarajan, and B. P. Pal, "Conception, realization, and characterization of a very high negative chromatic dispersion fiber," Optical Fiber Technology 8, 89-105 (2002).

5. F. Gerome, J.-L. Auguste, J. Maury, J.-M. Blondy, and J. Marcou, "Theoretical and experimental analysis of a chromatic dispersion compensating module using a dual concentric core fiber," Journal of Lightwave Technology 24, 442-448 (2006).

6. H. Subbaraman, T. Ling, Y. Jiang, M. Y. Chen, P. Cao, and R. T. Chen, "Design of a broadband highly dispersive pure silica photonic crystal fiber," Applied Optics 46, 3263-3268 (2007).

7. M. Bendsøe and N. Kikuchi, "Generating optimal topologies in structural design using a homogenization method," Computational methods in Applied Mechanics and Engineering 71, 197-224 (1988).

8. M. Bendsøe and O. Sigmund, "Topology Optimization: Theory, Methods and applications," Springer (2003).

9. U. D. Larsen, O. Sigmund and S. Bouwstra, "Design and fabrication of compliant micromechanisms and structures with negative poisson's ratio," IEEE Journal of Microelectromechanical Systems 6, 99-106 (1997).

10. T. Borrvall and J. Petersson, "Topology Optimisation of Fluids in Stokes Flow," International Journal for Numerical Methods in Fluids 41, 77-107 (2003).

11. J. S. Jensen and O. Sigmund, "Systematic design of photonic crystal structures using topology optimisation: low-loss waveguide bends," Applied Physics Letters 84, 2022-2024 (2004).

12. J. S. Jensen and O. Sigmund, "Topology optimization of photonic crystal structures: A high bandwidth low loss T-junction waveguide," Journal of the Optical Society of America B 22, 69-71 (2005).

13. P. I. Borel, A. Harpøth, L. H. Frandsen, M. Kristensen, J. S. Jensen, P. Shi and O. Sigmund, "Topology optimization and fabrication of photonic crystal structures," Optics Express 12, 1996-2001 (2004). 
14. A. Bjarklev, J. Broeng and A. S. Bjarklev "Photonic Crystal Fibres," Kluwer Academic Publishers (2003)

15. T. Fujisawa, K. Saitoh, K. Wada and M. Koshiba "Chromatic dispersion profile optimisation of dual-concentric-core photonic crystal fibers for broadband dispersion compensation," Optics Express 14, 893-900 (2006)

16. F. Poletti, V. Finazzi, T. M. Monroe, N. G. R. Broderick, V. Tse and D. J. Richardson, "Inverse design and fabrication tolerances of ultra flattened dispersion holey fibres," Optics Express 13, 3728-3736 (2005)

17. O. Sigmund and J. Petersson,"Numerical instabilities in topology optimization: A survey on procedures dealing with checkerboards, mesh-dependencies and local minima," Structural Optimization 16, 68-75 (1998).

18. J. Riishede, N.A. Mortensen and J. Lægsgaard, "A poor man's approach to modelling microstructured optical fibres," Journal of Optics A: Pure and Applied Optics 13, 534538 (2003).

19. T. E. Bruns and D. A. Tortorelli, "Topology optimization of non-linear elastic structures and compliant mechanisms," Computer Methods in Applied Mechanics and Engineering 190, 3443-3459 (2001).

20. B. Bourdin, "Filters in topology optimization," International Journal for Numerical Methods in Engineering 50, 2143-2158 (2001).

21. K. Svanberg, "The method of moving assymptotes - a new method for structural optimization," International Journal for Numerical Methods in Engineering 24, 359-373 (1987).

22. www.corning.com/opticalfiber

23. A. P. Seyranian, E. Lund, and N. Olhoff, "Multiple eigenvalues in structural optimization problems," Structural Optimization 8, 207-227 (1994).

24. J. S. Jensen and N. L. Pedersen, "On maximal eigenfrequency separation in two-material structures: the 1D and 2D scalar cases," Journal of Sound and Vibration 289, 967-986 (2006). 


\section{Figure Captions}

Fig. 1. A schematic illustration of the different steps of the topology optimization method.

Fig. 2. Geometry of the density filtering function, $s$, for increasing values of the filter width, $w$. Only filter functions with an uneven number of grid points along the diameter are used.

Fig. 3. Comparison of the dispersion of the optimized DCF design, and the optimization target corresponding to $-5 \times$ the dispersion of a standard single-mode fibre.

Fig. 4. Spatial distribution of the refractive index for the optimized DCF design.

Fig. 5. Cross section of the index profile along the diameter of the calculation domain. A density filter width of $\mathrm{w}=5$ has been applied. b) The same calculation as in figure a, but using a filter width of $\mathrm{w}=1$, i.e. disabling the density filter.

Fig. 6. The refractive index profile of an optimized DCF design obtained by using a single-mode constraint. A density filter width of $w=5$ has been applied during the optimization.

Fig. 7. The effective mode indices of the LP01, LP11 and LP02-mode as a function of the iteration number. The single-mode constraint is seen to force the mode index of the higherorder modes below the background index thus keeping the optimized design single-moded. 


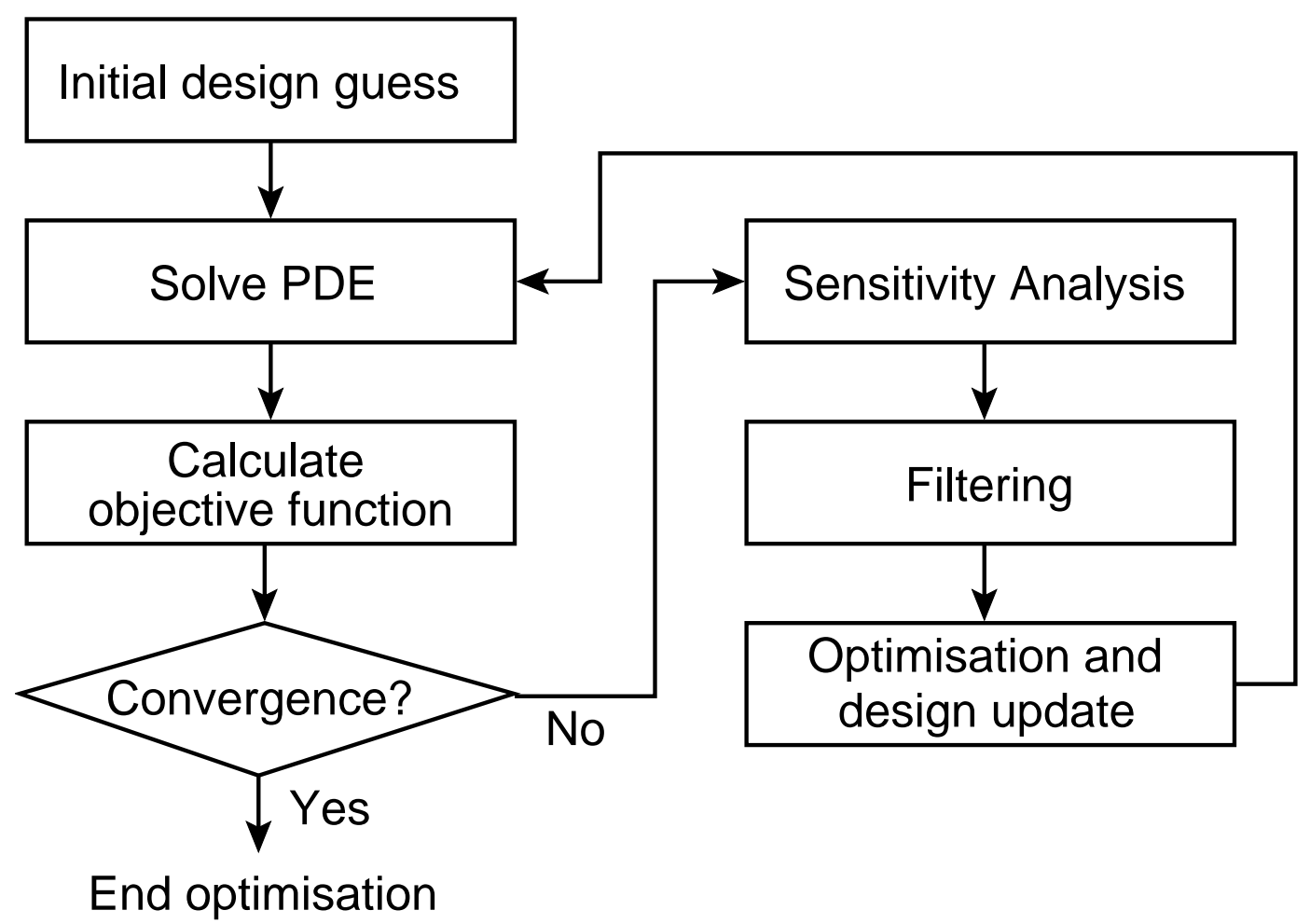

Fig. 1. A schematic illustration of the different steps of the topology optimization method. 
$\mathbf{w}=1$

$\mathbf{w}=3$

$\mathrm{w}=5$
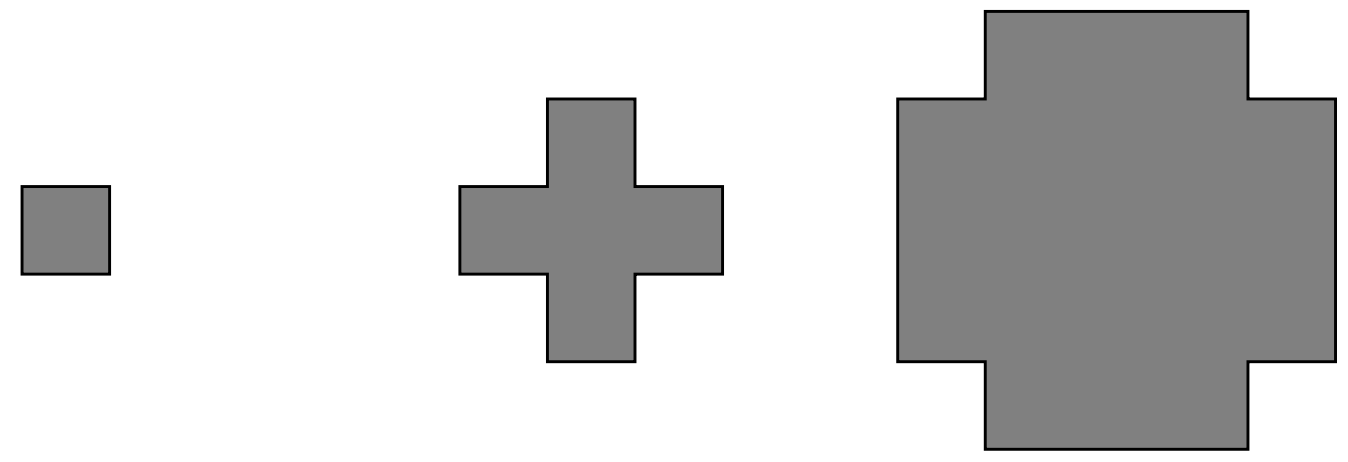

$\square s=1$

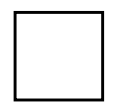

$\mathrm{s}=0$

Fig. 2. Geometry of the density filtering function, $s$, for increasing values of the filter width, $w$. Only filter functions with an uneven number of grid points along the diameter are used. 


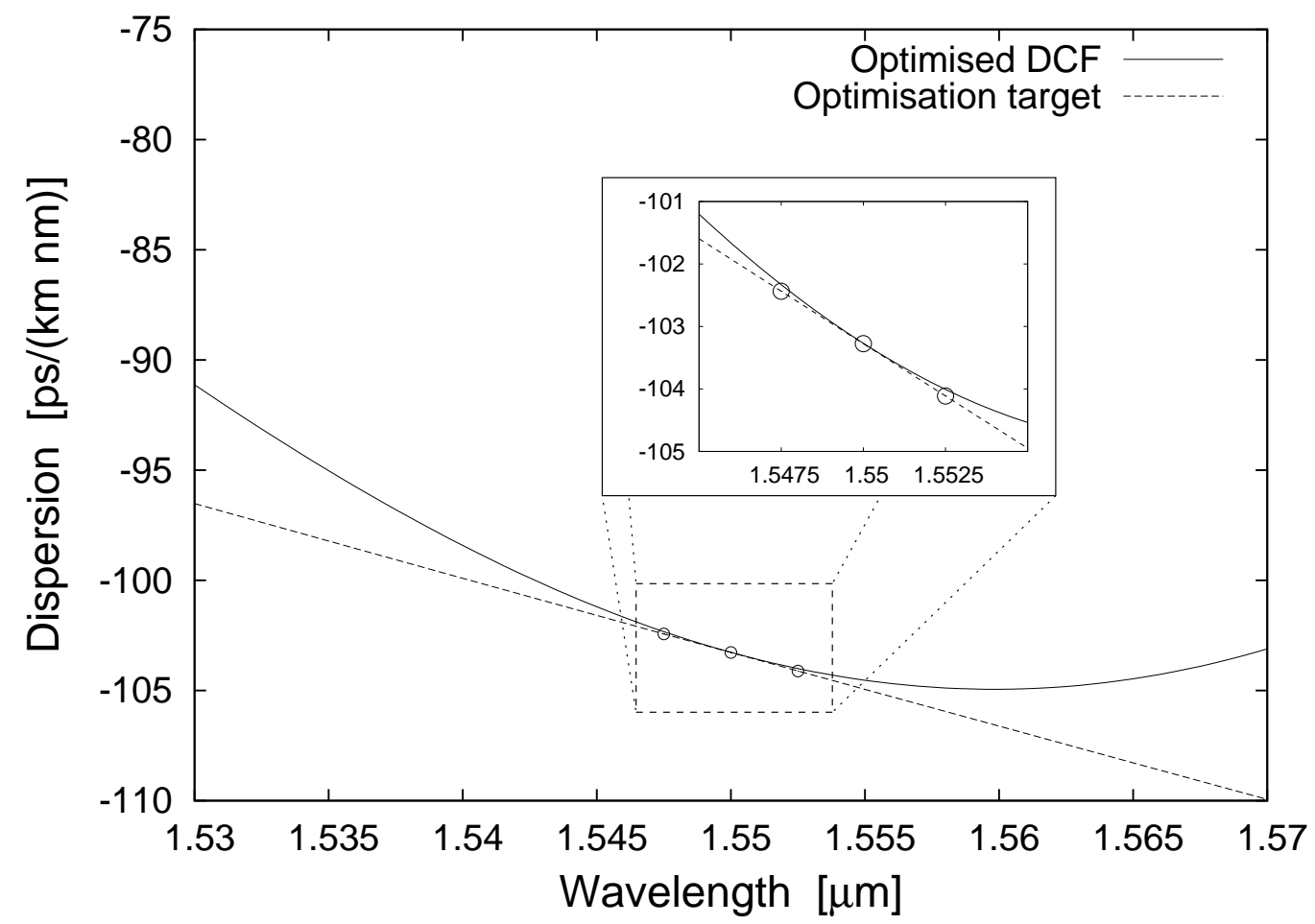

Fig. 3. Comparison of the dispersion of the optimized DCF design, and the optimization target corresponding to $-5 \times$ the dispersion of a standard singlemode fibre. 


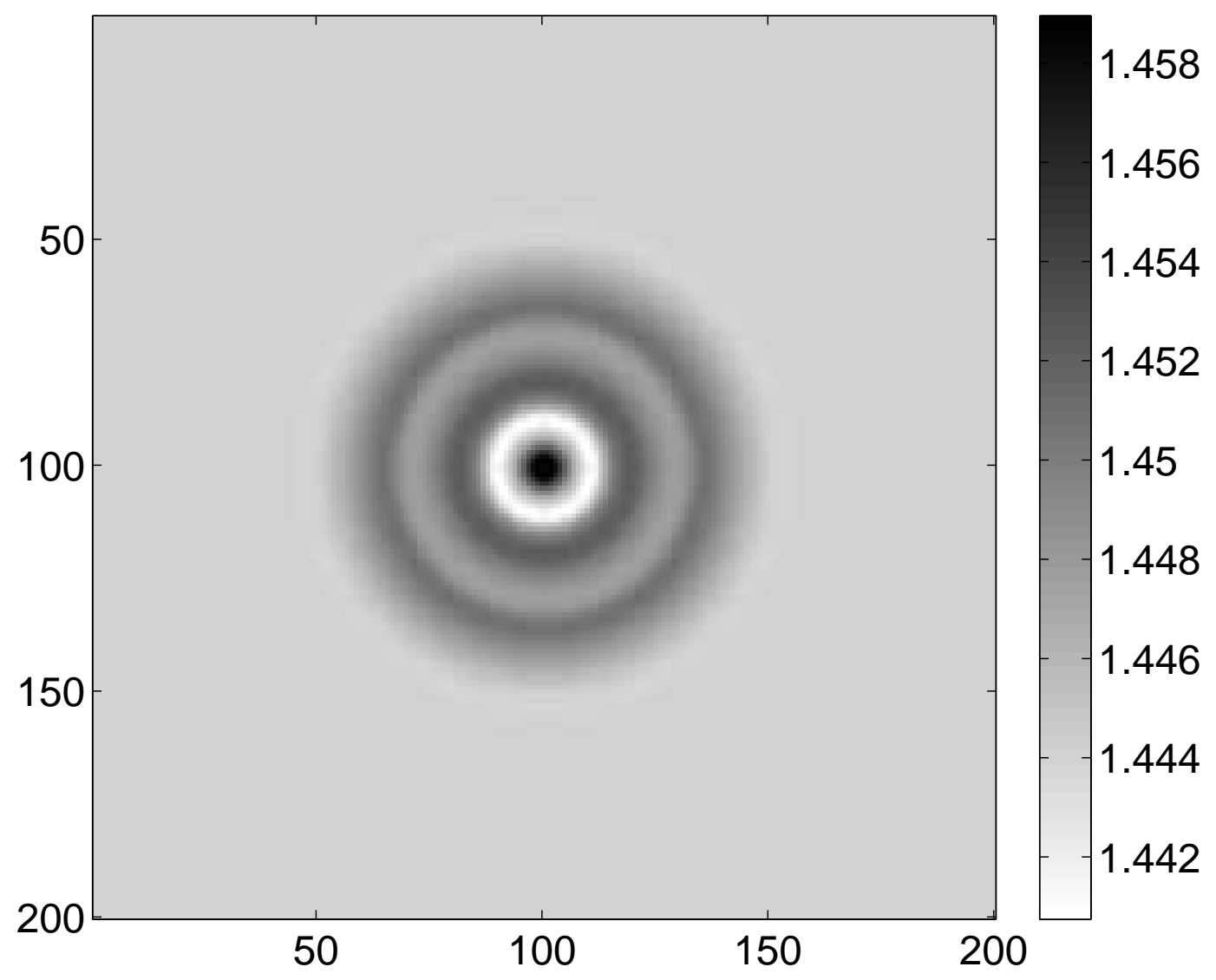

Fig. 4. Spatial distribution of the refractive index for the optimized DCF design. 

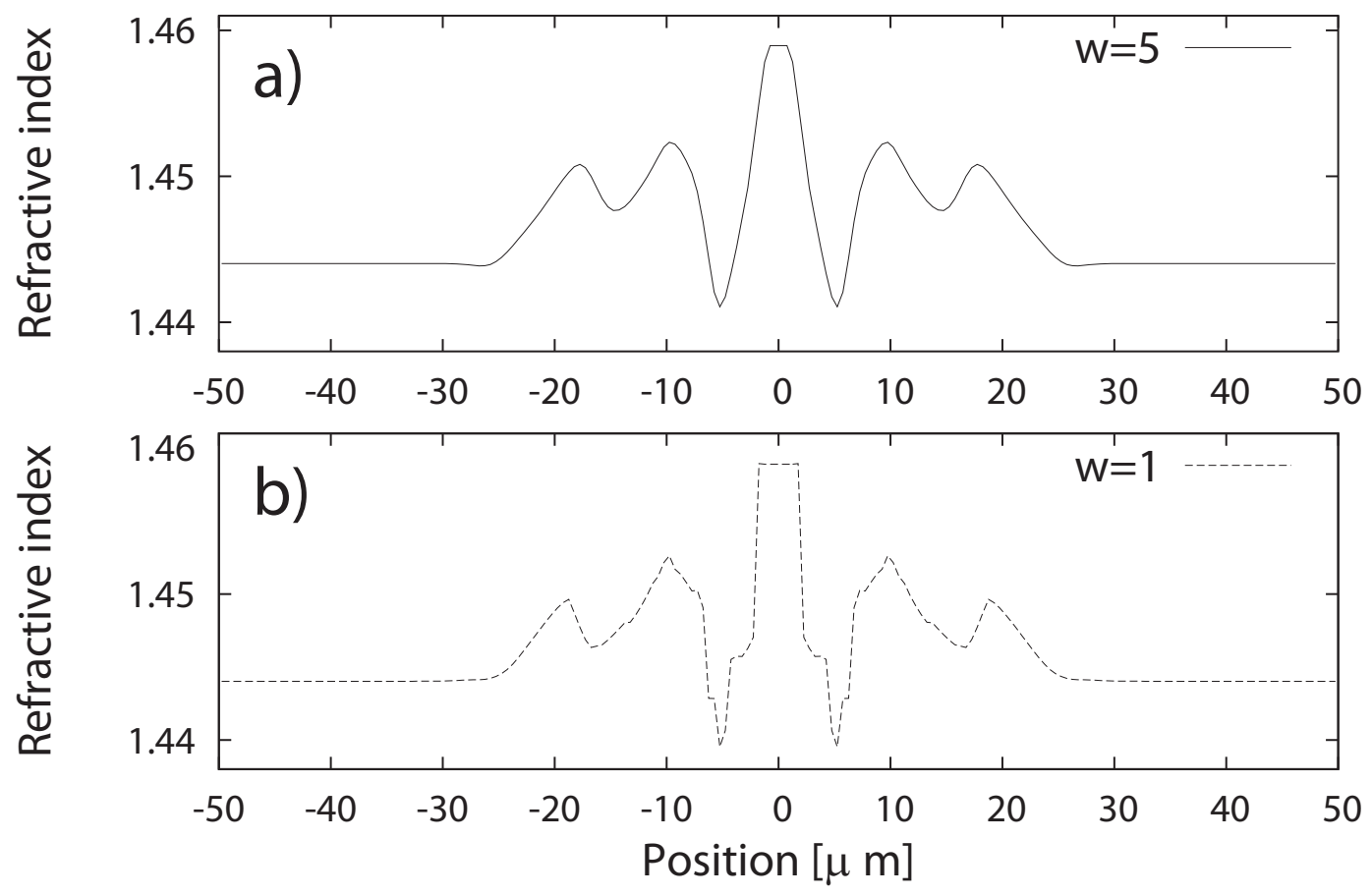

Fig. 5. a) Cross section of the index profile along the diameter of the calculation domain. A density filter width of $\mathrm{w}=5$ has been applied. b) The same calculation as in figure a, but using a filter width of $\mathrm{w}=1$, i.e. disabling the density filter. 


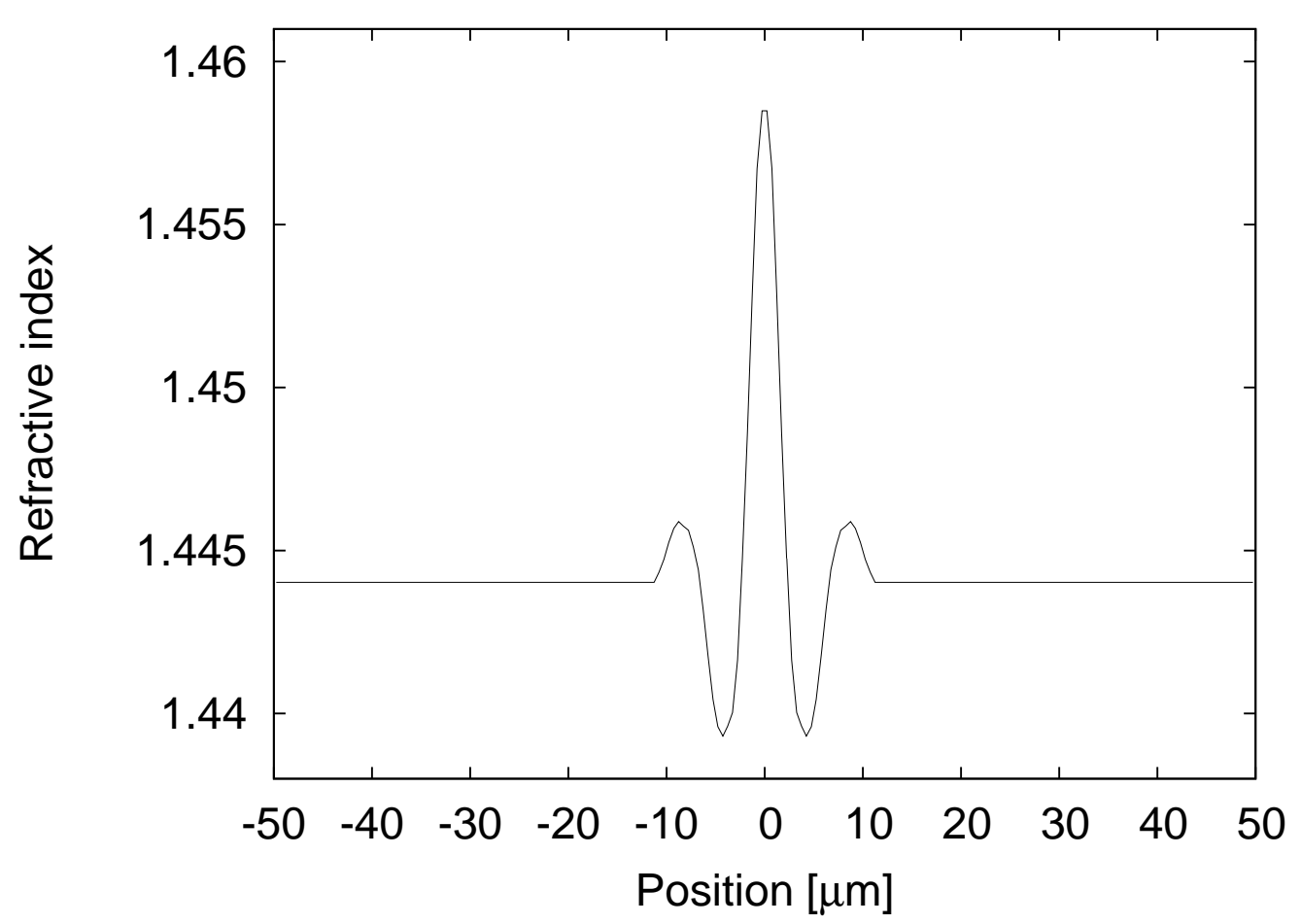

Fig. 6. The refractive index profile of an optimized DCF design obtained by using a single-mode constraint. A density filter width of $w=5$ has been applied during the optimization. 


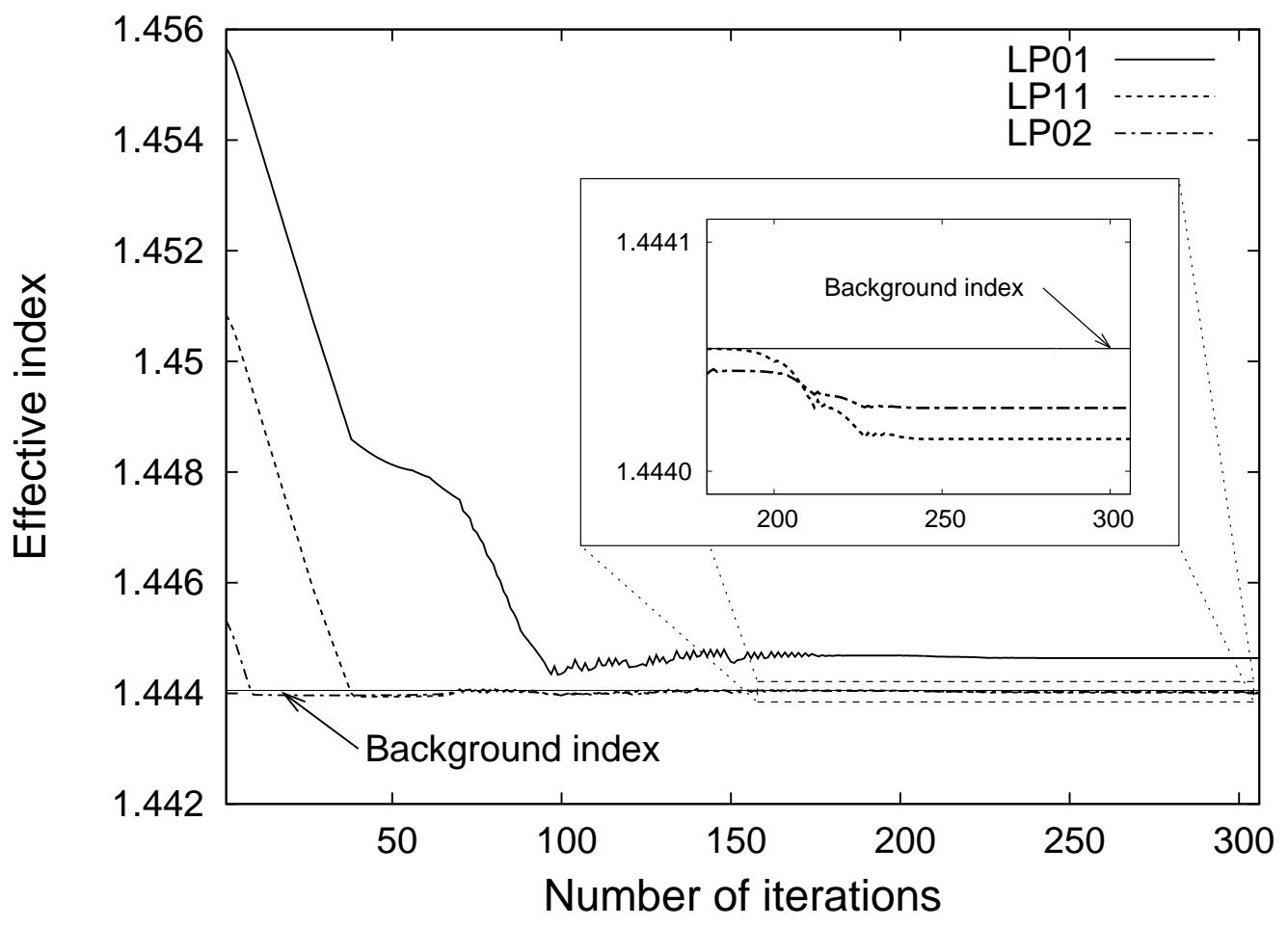

Fig. 7. The effective mode indices of the LP01, LP11 and LP02-mode as a function of the iteration number. The single-mode constraint is seen to force the mode index of the higher-order modes below the background index thus keeping the optimized design single-moded. 